\title{
1 Diverse functions associate with trans-species polymorphisms in humans
}

3 Keila Velázquez-Arcelay ${ }^{1}$, Mary Lauren Benton ${ }^{2}$, and John A. Capra ${ }^{1,3,4^{*}}$

4

$5 \quad{ }^{1}$ Department of Biological Sciences, Vanderbilt University

$6 \quad{ }^{2}$ Department of Computer Science, Baylor University

$7 \quad{ }^{3}$ Departments of Biomedical Informatics and Computer Science, Genetics Institute, and Center for Structural

8 Biology, Vanderbilt University

$9 \quad{ }^{4}$ Bakar Computational Health Sciences Institute and Department of Epidemiology and Biostatistics, University of

10 California, San Francisco

$11{ }^{*}$ Corresponding author: tony.capra@ucsf.edu

\section{Abstract}

14 Long-term balancing selection (LTBS) can maintain allelic variation at a locus over millions of

15 years and through speciation events. Variants shared between species, hereafter "trans-species

16 polymorphisms" (TSPs), often result from LTBS due to host-pathogen interactions. For instance,

17 the major histocompatibility complex (MHC) locus contains TSPs present across primates. Several

18 hundred TSPs have been identified in humans and chimpanzees; however, because many are in

19 non-coding regions of the genome, the functions and adaptive roles for most TSPs remain

20 unknown. We integrated diverse genomic annotations to explore the functions of 125 previously

21 identified non-coding TSPs that are likely under LTBS since the common ancestor of humans and

22 chimpanzees. We analyzed genome-wide functional assays, expression quantitative trait loci

23 (eQTL), genome-wide association studies (GWAS), and phenome-wide association studies

24 (PheWAS). We identify functional annotations for 119 TSP regions, including 71 with evidence

25 of gene regulatory function from GTEx or genome-wide functional genomics data and 21 with 
26 evidence of trait association from GWAS and PheWAS. TSPs in humans associate with many

27 immune system phenotypes, including response to pathogens, but we also find associations with a

28 range of other phenotypes, including body mass, alcohol intake, urate levels, chronotype, and risk-

29 taking behavior. The diversity of traits associated with non-coding human TSPs suggest that

30 functions beyond the immune system are often subject to LTBS. Furthermore, several of these trait

31 associations provide support and candidate genetic loci for previous hypothesis about behavioral

32 diversity in great ape populations, such as the importance of variation in sleep cycles and risk

33 sensitivity.

35 Keywords: balancing selection; long-term balancing selection; trans-species polymorphisms;

36 phenome-wide association study

\section{Significance statement}

39 Most genetic variants present in human populations are young $(<100,000$ years old); however, a

40 few hundred are millions of years old with origins before the divergence of humans and

41 chimpanzees. These trans-species polymorphisms (TSPs) were likely maintained by balancing

42 selection - evolutionary pressure to maintain genetic diversity at a locus. However, the functions

43 driving this selection, especially for non-coding TSPs, are largely unknown. We integrate

44 genome-wide annotation strategies to demonstrate TSP associations with immune system

45 function, behavior (addition, cognition, risky behavior), uric acid metabolism, and many other

46 phenotypes. These results substantially expand our understanding of functions TSPs and suggest

47 a substantial role for balancing selection beyond the immune system. 
Introduction

51 The interaction between populations and environments is dynamic. Over time, allele frequencies

52 in a population shift due to drift and adaptive responses to specific environmental pressures.

53 Most genetic variants are short-lived compared to the timescale of species. But on rare occasions

54 variants persistently segregate at intermediate frequencies for millions of years, sometimes

55 predating the most recent common ancestor (MRCA) between two sister species (Bitarello et al.,

56 2018; Cheng \& DeGiorgio, 2019; DeGiorgio, Lohmueller, \& Nielsen, 2014; Leffler et al., 2013;

57 Siewert \& Voight, 2017; Teixeira et al., 2015). These trans-species polymorphisms (TSPs) are

58 likely a sign of genomic regions under long-term balancing selection (LTBS). Over time,

59 instances of LTBS leave signatures in the genome that differentiate them from those under other

60 forms of selection (Bitarello et al., 2018; Key, Teixeira, de Filippo, \& Andrés, 2014; Leffler et

61 al., 2013; Siewert \& Voight, 2017).

Several instances of likely LTBS have been observed in humans and other primates,

63 mostly within the major histocompatibility complex (MHC) or the ABO blood group locus. For

64 example, the MHC, or human leukocyte antigen (HLA) system in humans, is a family of varied

65 proteins expressed on the cell surface with essential functions in adaptive immune response and

66 regulation. Balancing selection on different components of the HLA region dates to the common

67 ancestor between chimpanzees and humans (Azevedo, Serrano, Amorim, \& Cooper, 2015).

68 Similarly, the ABO gene has three alleles, and its variants lead to different blood cell antigens, or

69 lack of thereof, on the surface of the cell. Variation in this group could have a benefit in the

70 immune response to pathogens, and balanced polymorphisms at this locus date back to the

71 common ancestor of gorillas, orangutans, and humans (Ségurel et al., 2012). Other immune- 
72 related genes show LTBS between humans and other primates, e.g.: TRIM5, a RING finger

73 protein 88 (Battivelli et al., 2011; Cagliani et al., 2010; Ganser-Pornillos \& Pornillos, 2019), and

74 ZC3HAV1, a zinc finger CCCH-type antiviral protein 1 (Cagliani et al., 2012; De Filippo et al.,

75 2016; Mao et al., 2013; Todorova, Bock, \& Chang, 2015). These genes have important roles in

76 host/pathogen response through inhibition of virus replication. can also be beneficial when a population needs to adapt to new environments. Some variants

79 found under balancing selection in Africa have come to be under directional selection in non-

80 African populations (European and Asian), with one allele becoming predominant in the

81 population (De Filippo et al., 2016). This suggests the adaptive potential of the variation

82 maintained under balancing selection; however, most of these variants were not likely under

83 LTBS.

Recent studies have developed tools and methods to identify instances of balancing selection in genome-wide data (Siewert \& Voight, 2017, 2020). Leffler et al. (2013) compared

86 polymorphisms across the genome in Yoruba individuals from the 1000 Genomes Project to

87 those found in Western chimpanzees sequenced by the PanMap Project. They identified coding

88 variants in 324 genes and 125 non-coding haplotypes with multiple TSPs suggesting the

89 presence of LTBS.

90 Despite the importance and prevalence of balancing selection, most regions bearing

91 signatures of LTBS, including these TSPs, have not been functionally characterized.

92 Determining the functional roles of these polymorphisms in human adaptation and health will

93 deepen our understanding of the dynamics of balancing and positive selection and their roles in

94 adaptation to new environments. Here, we focus on the non-coding TSPs identified by Leffler et 
95 al. (2013). We identify potential functional drivers of the LTBS in humans by applying several

96 genome-wide functional annotations and association tests to these TSPs. Our results identify

97 diverse functions, including effects unrelated to the immune system, that may underlie LTBS on

98 the human and chimpanzee lineages.

$100 \quad$ Results

101 Human-chimpanzee TSPS

102 We consider 125 human genomic regions containing multiple TSPs that are segregating in both

103 humans and chimpanzees. This set is composed of 263 variants with strong evidence of identity-

104 by-descent; i.e., the divergence between the ancestral and derived alleles is deeper than the

105 human-chimp speciation event. These TSPs were identified by Leffler et al. (2013) based on the

106 observation from coalescent theory (Ségurel et al., 2012) that pairs of TSPs within $4 \mathrm{~kb}$ in the

107 human genome are extremely unlikely to result from neutral processes, and thus are strong

108 candidates for LTBS (Figure 1). Hereafter, we refer to these as "TSP regions".

109 In the following, we analyze two sets of variants for the 125 TSP regions. First, we focus

110 on the 263 TSPs themselves. The average distance between TSPs defining a region is $1652.7 \mathrm{bp}$

111 (standard deviation $=1303.2$ ). Second, to capture functions tagged by variants in high linkage

112 disequilibrium (LD) with TSPs, we also considered potential tag SNPs in high LD ( $\left.\mathrm{R}^{2} \geq 0.8\right)$ with

113 TSPs in African, European, and East Asian populations from the 1000 Genomes Project. This

114 LD-expanded set includes 10,259 variants across the 125 TSP regions (Supplementary Figure 1).

115 By expanding to include LD, we capture additional associations, but may also introduce false

116 positives; thus, we report results on both sets throughout. 


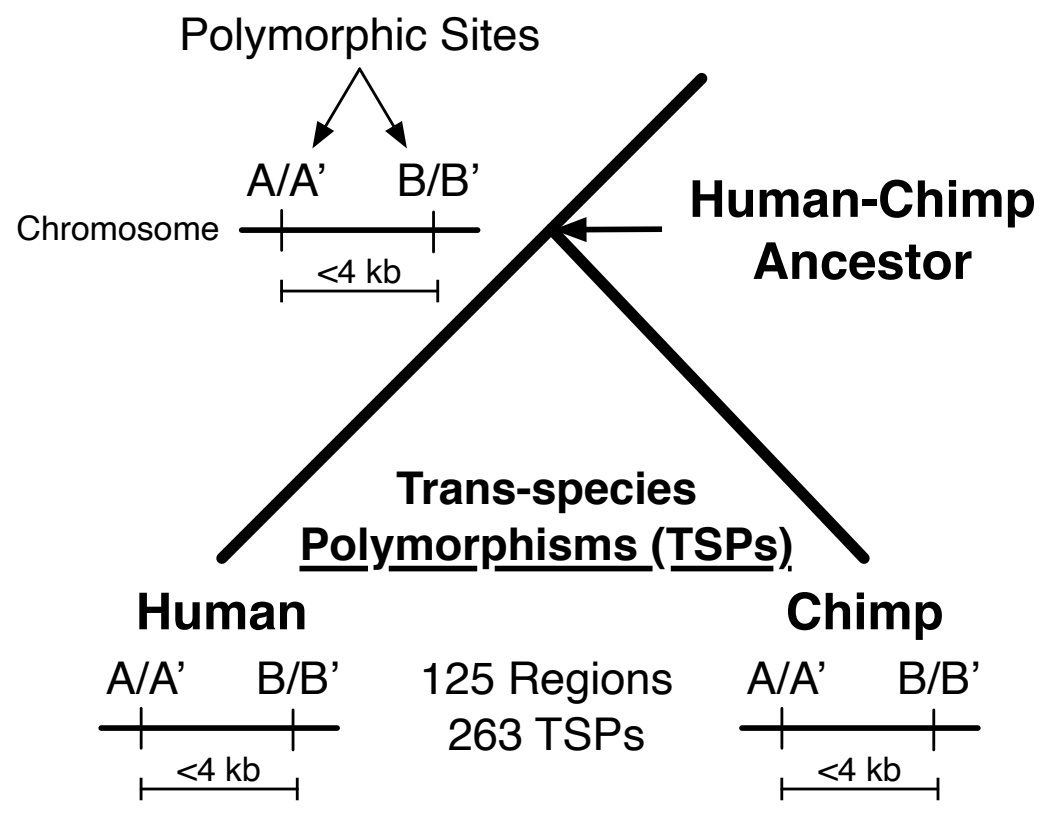

118 Figure 1. Trans-species polymorphisms (TSPs) likely resulting from long-term balancing

119 selection (LTBS). Schematic showing the criteria used by Leffler et al. (2013) to identify TSPs

120 likely maintained by LTBS. Each line represents a chromosome with polymorphisms segregating

121 in a population. $\mathrm{A} / \mathrm{A}^{\prime}$ are two alleles segregating in both humans and chimpanzees at one site

122 (i.e., a TSP), and B/B' are alleles segregating in both species at a nearby site. TSPs are very

123 unlikely to appear nearby (within $4 \mathrm{~kb}$ ) without the action of balancing selection. We consider

124125 TSP regions containing 263 TSPs. Within these regions, multiple functional scenarios are

125 possible. For example, one TSP may be under LTBS while the other is neutral, but maintained

126 due to tight linkage. Alternatively, the TSPs may have epistatic functions and both be under

127 selection. In addition to the 263 TSPs, we also considered functional associations with 9,996

128 variants in high LD $\left(\mathrm{r}^{2}>0.8\right)$ with a TSP at least one population from the 1000 Genomes Project

129 (Supplementary Figure 1). 


\section{Trans-species polymorphisms overlap diverse functional annotations}

132 We intersected the TSPs with diverse lines of functional evidence from large-scale genomic

133 studies, including genome-wide functional genomics assays, eQTL, GWAS, and PheWAS. We

134 found at least one functional annotation for 95\% of the TSP regions (119 out of 125) covering

135130 TSPs and 4,807 LD SNPs (Figure 2). Here, we provide an overview of the overlap with

136 these annotations. In future sections, we provide details about each of these annotations. Given

137 that these regions were filtered by Leffler et al. (2013) to exclude coding TSPs, variants in $91 \%$

138 (114 out of 125) of regions overlap annotated gene regulatory regions. This includes 58 TSPs in

13940 regions and 1334 LD variants in 112 regions. We also found 86 TSPs across 51 regions with

140 evidence of being expression quantitative trait loci (eQTL) in 29 tissues. Including the variants in

141 LD with TSPs, $57.6 \%$ of the regions (72 out of 125) contain eQTLs (p < E-5) in 49 tissues. We

142 found genome-wide significant associations with phenotypes in available genome- or phenome-

143 wide association studies for $19 \%$ of the regions (24 out of 125; 13 GWAS and 16 geneAtlas

144 PheWAS). Finally, 11.2\% (14 out of 125) of these regions contain SNPs in protein-coding

145 regions; two TSPs in different regions produce non-synonymous protein sequence changes, and

14617 other non-synonymous variants in 7 regions are in high LD with the TSPs. (These were not

147 identified as coding in the previous study due to changes in protein annotations.) 


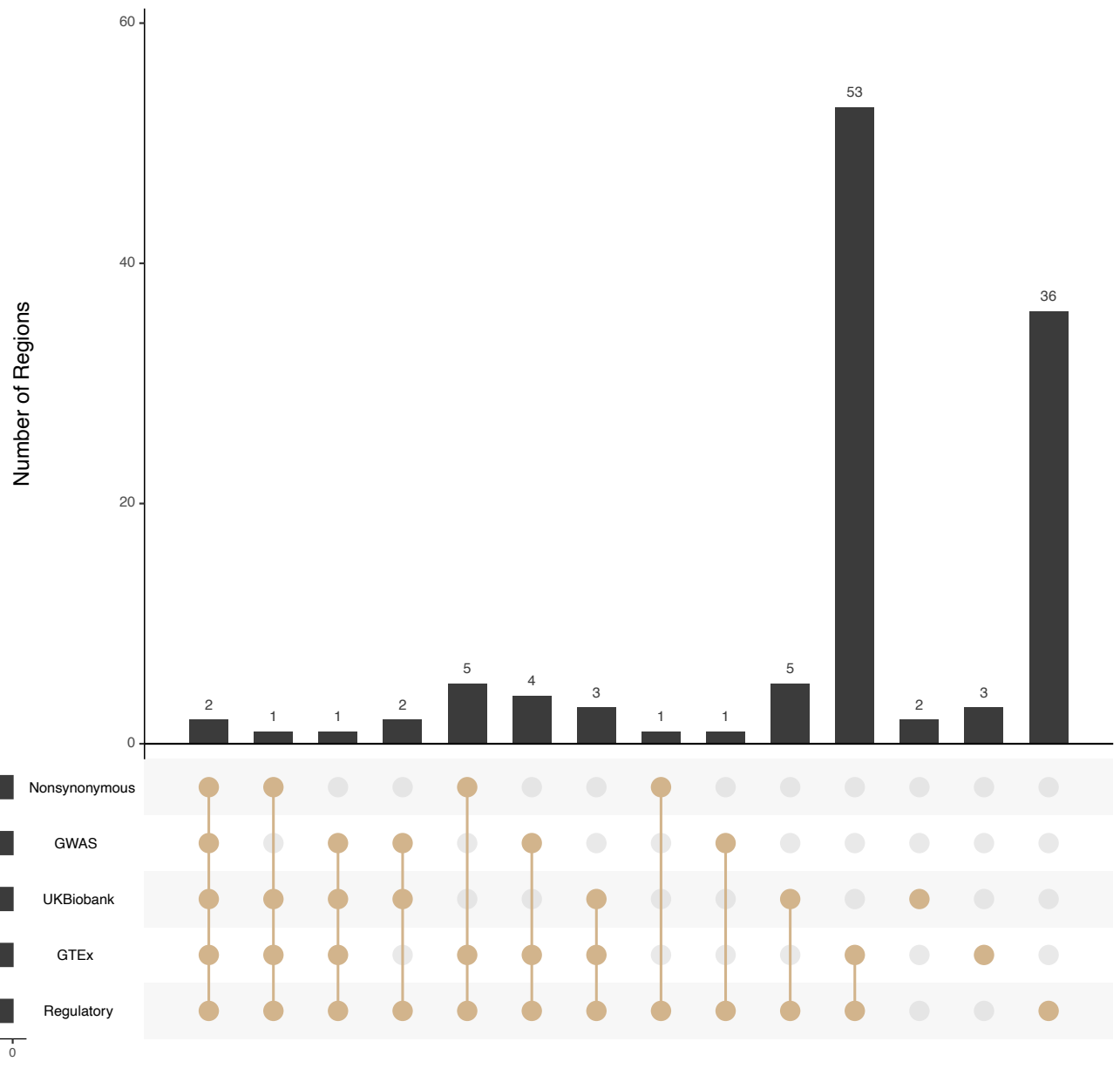

150 Figure 2. Functional annotations available for the expanded TSP regions. Summary of the

151 annotations of each type available for TSP regions, including tagging variants in high LD with

152 TSPs. A total of 119 out of 125 TSP regions contain at least one line of functional evidence.

153 Multiple lines (two or more) are available for 78 regions.

155 Evidence of gene regulatory function for TSPS

156 We hypothesized that many of the non-coding TSPs in our set perform gene regulatory

157 functions. To evaluate this possibility, we intersected the TSPs and variants in high LD with

158 maps of functional regulatory regions from the Ensembl regulatory build (Zerbino, Wilder,

159 Johnson, Juettemann, \& Flicek, 2015). We found 58 TSPs with regulatory annotations in 40 TSP 
160 regions, and additionally $1334 \mathrm{LD}$ variants in 114 regions. These include variants in CTCF

161 binding sites, open chromatin regions, promoter flanking regions, enhancers, promoters, and

162 known TF binding sites. Supplementary table 1

163 Overlap of a variant with a regulatory annotation does not necessarily imply a regulatory

164 function. To consider additional evidence of regulatory function, we examined eQTL in 50

165 GTEx tissues for overlap with TSPs. At least one eQTL was found for 51 of the TSP regions

166 (40\%). Among these 51 regions, 29 TSPs are themselves eQTL; for the remainder, variants in

167 high LD with TSPs were eQTL. The eQTL were found across diverse tissues (Figure 3A), and

168 there was no enrichment for specific gene ontology (GO) terms among the set of genes

169 influenced by TSP eQTL. This suggests that the targets of balancing selection have functions in

170 gene regulation across diverse tissues beyond the immune system.

171 Next, we tested if the TSP regions are enriched for overlap with any specific types of

172 regulatory regions compared to the expected overlap if they were randomly distributed across the

173 genome. We shuffled the TSP regions 1,000 times maintaining their length and chromosome

174 distributions and avoiding genome assembly gaps and ENCODE blacklist regions and counted

175 the number of overlaps observed for each random permutation. The TSPs overlap slightly fewer

176 base pairs annotated with regulatory functions than expected by chance, with significant $(\mathrm{P}<$

177 0.05) depletion for promoter and CTCF sites (Figure 3B). Since variants in these regions are

178 likely to influence gene regulation in many tissues (e.g., compared to enhancers which are often

179 context-specific), this suggests that individual TSPs may be less pleiotropic than expected by

180 chance. 


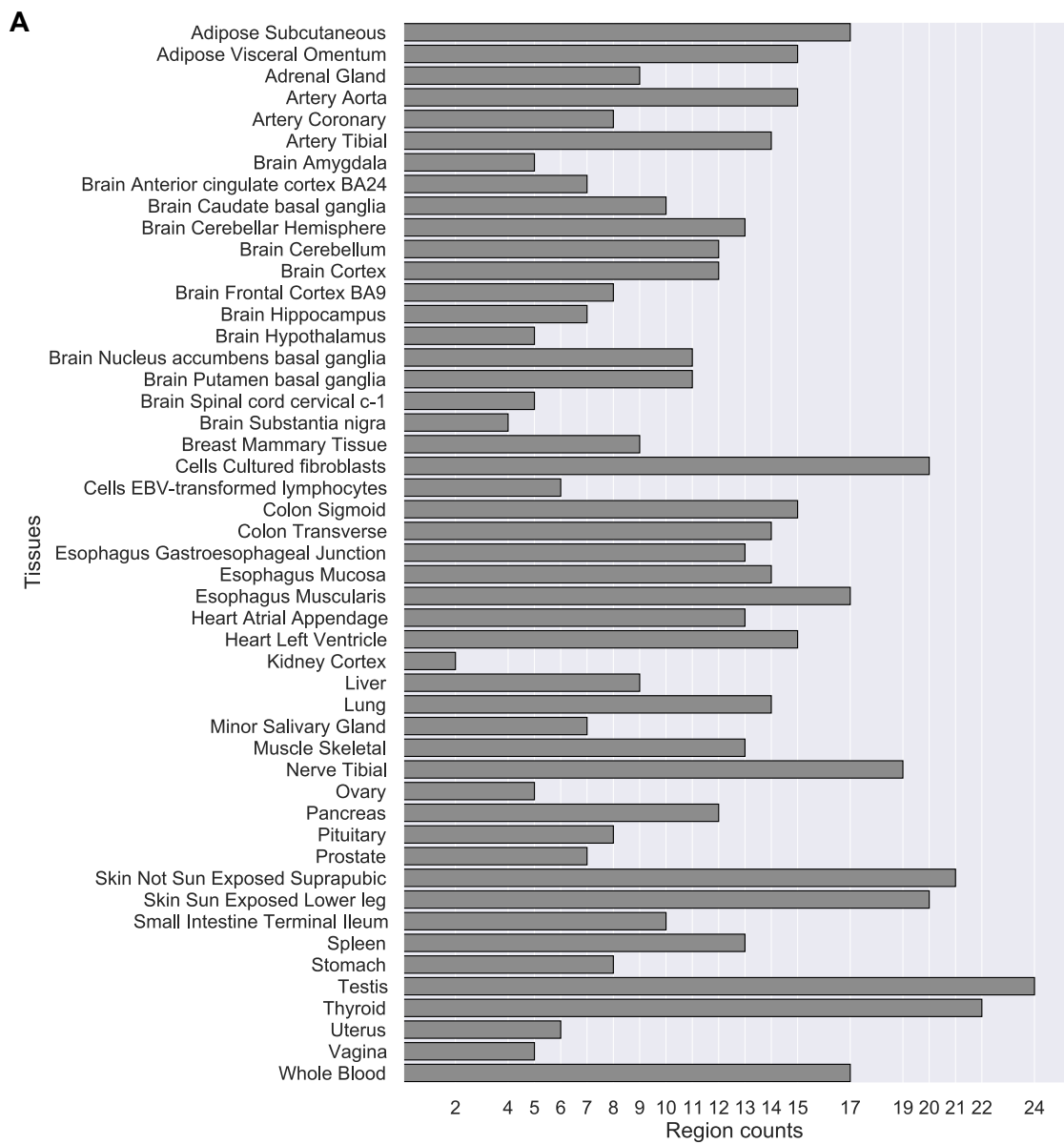

B

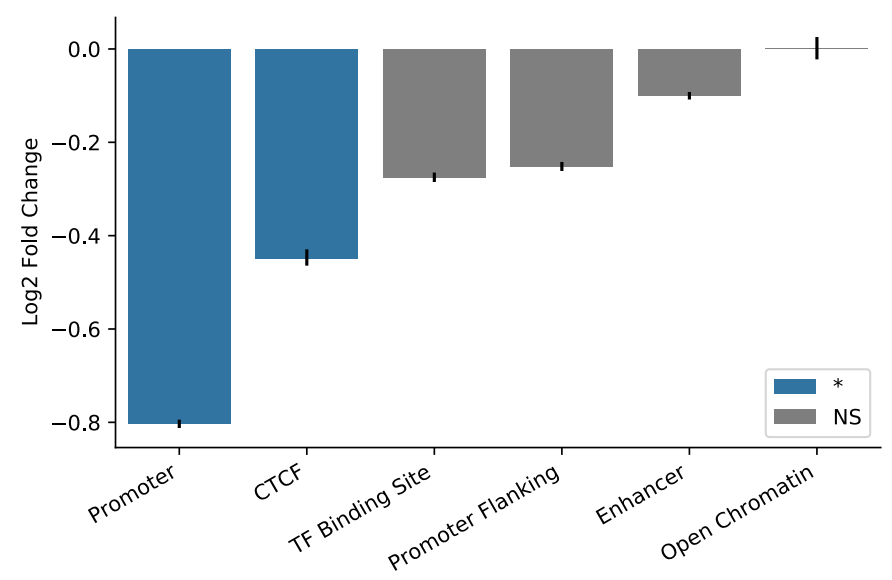

183 Figure 3. TSPs are eQTLs in diverse tissues and are depleted for overlap with promoters

184 and CTCF sites. (A) The number of TSP regions that contain an eQTL for each GTEx tissue.

185 Variation in TSP regions associates with gene expression in diverse tissues. The associated genes

186 also have Gene Ontology (GO) annotations from diverse functional categories (Supplementary 
187 Figure 2). (B) TSP regions are significantly depleted for overlap with promoters and CTCF sites

188 compared to length- and chromosome-matched non-coding regions from the genomic

189 background. The error bars represent $95 \%$ confidence intervals.

191 Genome-wide association studies link TSPs to traits

192 Genome-wide association studies have identified thousands of associations between genetic

193 variants and human traits. We intersected the TSP regions with associations reported in the

194 GWAS Catalog, which is currently composed of 227,262 associations. Since TSPs themselves

195 were not always directly tested in GWAS studies, we also include genome-wide significant $(\mathrm{p}<$

196 1E-8) associations with the tag variants in high LD with TSPs. We found significant associations

197 for 29 different variants (Figure 4; Supplementary Table 2). Two main functional categories

198 were identified in the GWAS associations for these variants: immunological functions and

199 neurological/behavioral traits. The associations with immune traits were expected given the

200 results of previous balancing selection studies and the few well-characterized instances of LTBS.

201 We identified many variants in LD with TSPs as associated with blood measurement phenotypes

202 and diseases related to immune response failure (chronic inflammatory diseases and ulcerative

203 colitis). We also found many neurological/behavioral related associations among these variants.

204 These traits include cognitive performance (PLCL1), chronotype (RP11-497E19.2), addiction

205 (alcohol use disorder, GPR139; smoking status, PLCL1), risky behavior (speeding propensity,

206 C3orf58), and mood swings (PLCL1). In addition to the immune response and neurological

207 categories, we observed associations with polycystic ovary syndrome, urate levels (HNF4G),

208 pancreatic cancer, hepatocyte growth factor levels, and gut microbiota (OTOS). We discuss

209 several of these associations in more detail in following sections. 
211 Phenome-wide association studies link TSPS to additional diverse traits

212 The growth of biobanks with linked genetic and phenotypic data has enabled the testing of the

213 association of genetic variants with diverse traits within a single cohort. This PheWAS approach

214 enables exploration of the functional and potentially pleiotropic effects of variants of interest

215 (Bush, Oetjens, \& Crawford, 2016). Using published associations from the UK Biobank, we

216 analyzed the association of TSPs with 778 traits; all 125 of the TSP regions were tested. Overall,

21721 TSPs in 16 regions had at least one genome-wide significant association (Figure 4, $\mathrm{P}<1 \mathrm{E}-8$ ).

218 Though testing different phenotypes than the GWAS, these associations were qualitatively

219 similar to the GWAS results, in that blood and immune system phenotypes had many

220 associations with TSPs, but the TSPs were also associated with a more diverse set of phenotypes.

221 We found associations in three major categories: immune response traits, body and physical

222 measurements, and neuropsychiatric traits related to addiction. We also observed associations

223 with many other phenotypes, for example: walking habits, heart disorders, renal and kidney

224 problems, pleural plaques, transient ischemic attack, cancerous tumor, and rupture of synovium

225 and tendon (Supplementary Table 3). 


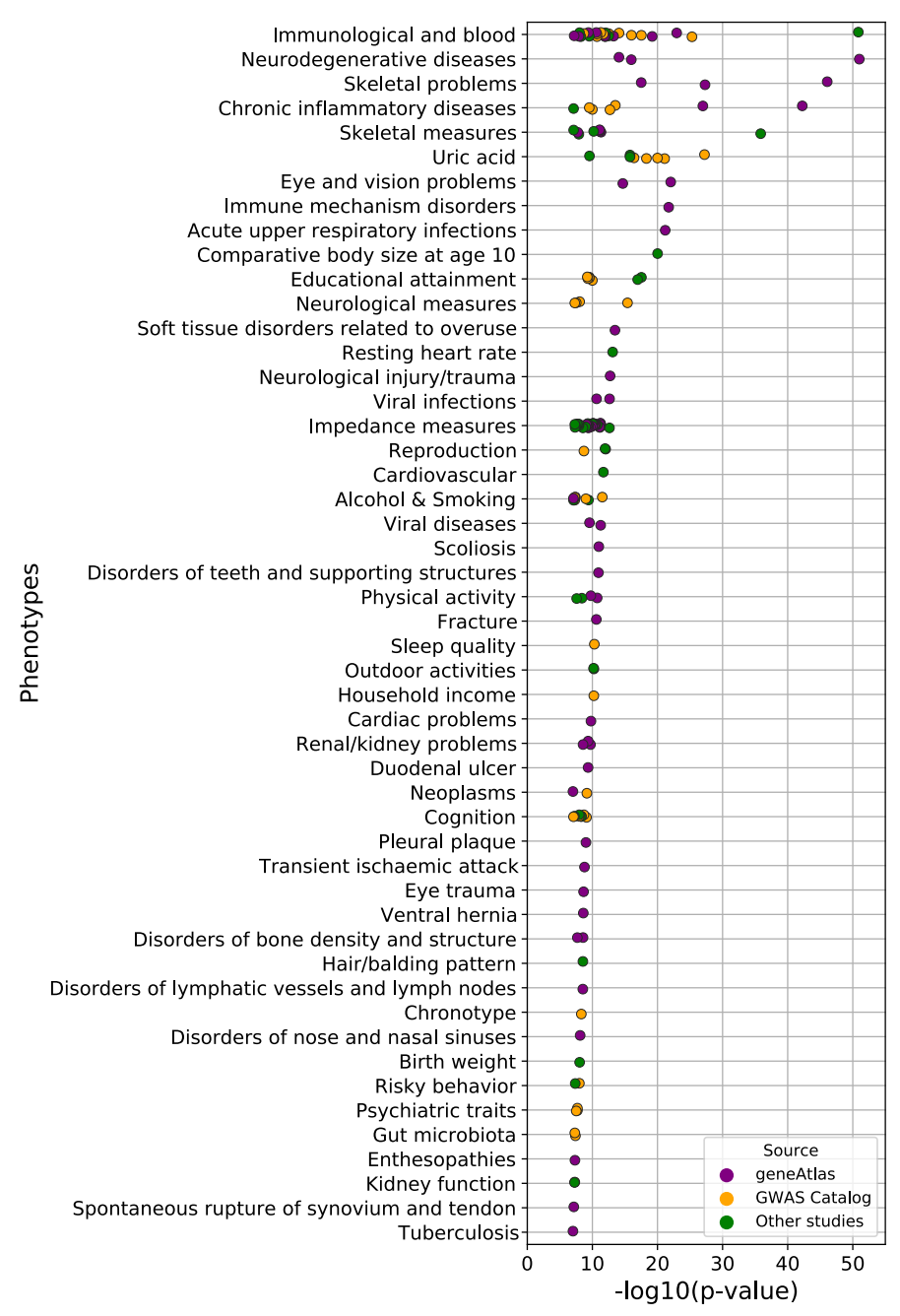

229 Figure 4. Genome- and phenome-wide association studies link TSPs to diverse traits.

230 Genome-wide significant $(\mathrm{P}<1 \mathrm{E}-8)$ associations from the GWAS Catalog (yellow) and from a

231 PheWAS over the UK Biobank from the geneAtlas (purple). Each dot represents an association

232 between a TSP region and a trait. Many immune-related traits are associated with TSPs, but there

233 are also associations with a wider variety of phenotypes including osseous, neurological, and

234 nervous system traits. Five extreme associations with immunological and blood traits $(\mathrm{P}<1 \mathrm{E}-$

235 60) were truncated for this visualization. Since few TSPs themselves were directly tested in the

236 GWAS Catalog, we include GWAS Catalog associations with tag variants in high LD $\left(\mathrm{r}^{2}>0.8\right)$ 
237 with TSPs. We also include results from other studies that published genome-wide results using

238 different cohorts.

240 Evidence of protein-coding function for TSPS

241 The TSPs were originally filtered by Leffler et al. (2013) to be non-coding; however, three of the

242263 TSPs are coding variants: a variant containing a synonymous allele in the gene dynein

243 (DNHD1) and another variant that results in a non-synonymous change in a transcript of

244 DNHD1's paralog DNAH9, and one non-synonymous variant in PKD1L2. This discrepancy is

245 due to changes in genomic annotations over time. For example, PKD1L2 is a calcium channel

246 with potential roles in kidney function. This gene is a polymorphic pseudogene in humans, with

247 the reference genome encoding the pseudogene form; this likely explains why this coding variant

248 was not identified as coding in previous studies. With the LD-expanded set, a total of 9 regions

249 had non-synonymous codon changes (19 variants, Supplementary Table 5). Some of the genes

250 containing these variants include: Sperm Associated Antigen 16 (SPAG1O), which codes for two

251 proteins that associate with the axoneme of sperm cells; Hepatocyte Nuclear Factor 4 Gamma

$252(H N F 4 G)$ codes for a receptor involved in DNA binding transcription activity; Leucine Rich

253 Colipase Like 1 ( $L R C O L 1)$ which has enzyme activation activity and is involved in digestion.

254 All of the coding variants had CADD scores suggestive of deleterious effects (scaled C-score >

255 20). Further work is needed to determine if these coding variants in high LD with the TSPs

256 influence selection.

258 Illustrative examples of diverse functions associated with TSP regions 
Integrating the above data, we found 79 TSP regions with two or more lines of functional

260 evidence. This included twelve regions with annotations from at least four sources. To illustrate

261 the diverse functions associated with TSPs, we highlight four of these regions.

263 Body mass and alcohol intake. A TSP (rs57790054) on 16p12.3 (hg38.chr16:19992138-

264 20043254) is strongly associated with several growth and body mass phenotypes as well as

265 alcohol intake frequency (Figure 5A; P $<4 \mathrm{E}-10$ for all). Another variant in high LD in

266 Europeans (rs72771074, $\left.\mathrm{R}^{2}=0.89\right)$ with a TSP (rs57790054) in this locus was associated with

267 alcohol use disorder in a previous GWAS in a European cohort $(\mathrm{P}=5 \mathrm{E}-8)$ (Sanchez-Roige et al.,

268 2019). The nearest gene, GPR139, encodes for a G-protein coupled receptor expressed in the

269 brain that is involved in alcohol drinking and withdrawal symptoms in rats (Kononoff et al.,

270 2018). This region contains several variants in LD with TSPs in regulatory regions, such as

271 CTCF binding sites (rs117293173, rs13338055, rs74011247, and rs79521770). One TSP

272 (rs57790054) is an eQTL for the gene KNOP1, and 26 other LD SNPs are eQTL for both

$273 K N O P 1$ and IQCK. These results suggest that effects on growth and BMI or on addictive

274 behaviors could be under LTBS. We note that there is some evidence of ethanol consumption in

275 chimpanzees, but it is unclear how widespread its availability was over the past several million

276 years (Hockings et al., 2015).

277

278 Urate levels. The TSPs (rs1839333, rs1913638) on 8q21.13 are both significantly associated (P <

279 2.0e-18) with uric acid levels in multiple GWAS in European and Asian ancestry populations

280 (Figure 5B) (Kanai et al., 2018; Köttgen et al., 2013; Tin et al., 2019). These variants are also

281 associated with a range of body mass traits in the UK Biobank. Another variant in this locus 
282 (rs2941471, $\mathrm{R}^{2}=0.97$ and $\mathrm{R}^{2}=0.82$ in East Asians and Europeans respectively) is associated with

283 pancreatic cancer ( $\mathrm{p}=7 \mathrm{E}-10)$. Though elevated uric acid in the blood is associated with many

284 conditions, it is a marker for pancreatic cancer (Stotz et al., 2014). This locus also contains two

285 non-synonymous variants in the gene $H N F 4 G$, a transcription factor expressed in the liver,

286 kidney, and pancreas, in high LD with the TSPs (rs1805098 and rs2943549). The TSPs are also

287 expression and splicing QTL for HNF4G (P = 1.9E-4 and 8.6E-6, respectively). Variants in

$288 H N F 4 G$ are associated with several traits, including the development of hyperuricemia (Chen et

289 al., 2017).

290 
bioRxiv preprint doi: https://doi.org/10.1101/2021.01.21.427090 this version posted January 22, 2021. The copyright holder for this preprint (which was not certified by peer review) is the author/funder, who has granted bioRxiv a license to display the preprint in perpetuity. It is made available under aCC-BY-NC 4.0 International license.

A

chr16:19992137-20054576

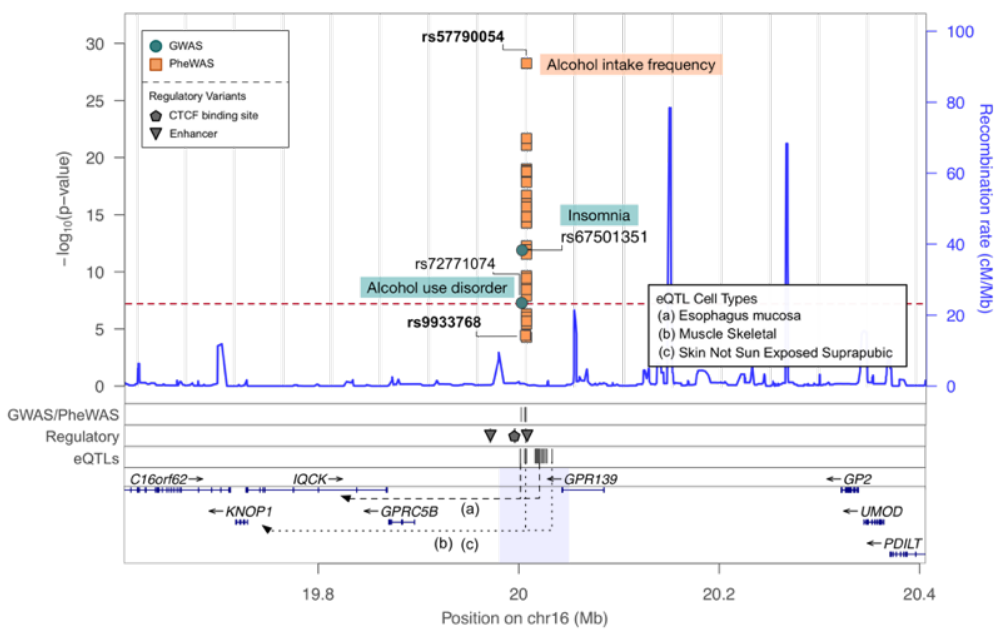

B

chr8:76442293-76564405

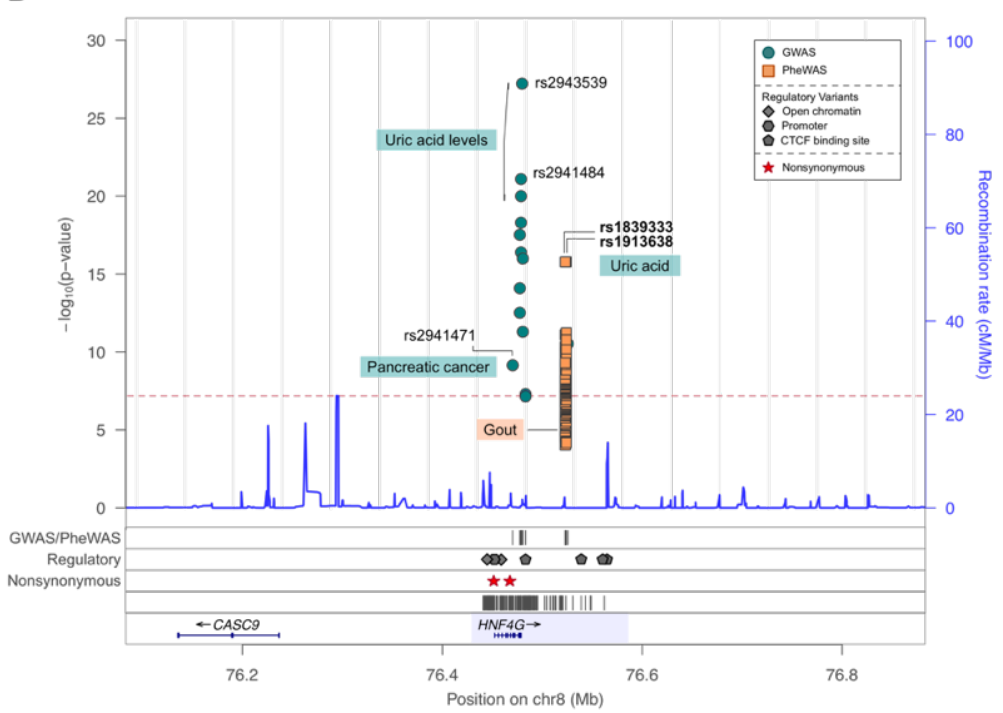

C

chr3:143636420-143740729

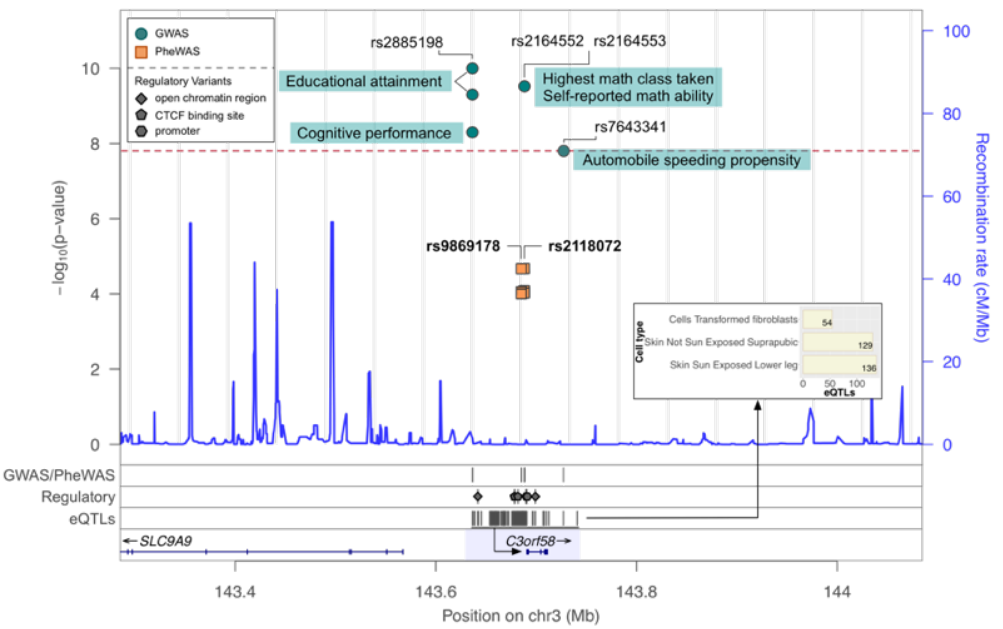

292 Figure 5. Illustrative examples of non-immune functions associated with TSPs. 
(A) A TSP in 16p12.3 is associated with body mass index (BMI) and alcohol intake. Regional association plot showing statistically significant genome- and phenome-wide associations

295 (threshold $\mathrm{p} \leq 1 \mathrm{E}-08$ ), regulatory annotations from Ensembl, and eQTLs from GTEx. One of the

296 TSPs (rs57790054, orange) is associated with alcohol intake and several growth and body mass

297 phenotypes in the UK Biobank. A variant in high LD (rs72771074, green) has been associated

298 with alcohol use disorder in a previous GWAS. The TSP is also strongly associated with growth

299 (comparative body size at age 10,9.6e-21) and body mass index (3.5e-12). The TSPs are nearby

300 GPR139, a gene encoding a G-protein coupled receptor expressed in the brain, whose expression

301 levels influence alcohol drinking behavior in rats. The TSP region also contains several CTCF

302 binding sites. TSP are shown in bold text.

303 (B) TSPs in 8q21.11 are associated with urate levels. Regional association plot showing

304 statistically significant genome- and phenome-wide associations ( $\mathrm{P} \leq 1 \mathrm{E}-08)$, eQTL, regulatory

305 and coding SNPs. LD SNPs in this region are associated with urate levels and pancreatic cancer.

306 A TSP (rs1839333) is also associated with gout, although the p-value did not meet our strict

307 threshold. TSP are shown in bold text. TSP are shown in bold text. Figures created using

308 LocusZoom (Pruim et al., 2011).

309 (C) TSP locus on 3q24. Regional association plot showing statistically significant genome- and

310 phenome-wide associations (threshold $\mathrm{p} \leq \mathrm{E}-08$ ), regulatory and eQTLs. This locus is

311 characterized by neurological traits involved in educational attainment, cognitive performance,

312 and risky behavior (automobile speeding propensity).

313

314 Chronotype. The TSPs (rs1887944, rs7147645) on 14q31.2 are both nominally associated (P <= 315 9.5e-6) with chronotype in a study of nearly 500,000 people (Jones et al., 2019). This is the only 
316 phenotype association with these variants. One variant in high LD (rs17119051) overlaps a

317 regulatory region, but there are no eQTL among the TSPs or other LD variants. Asynchrony in

318 chronotypes in a population has been proposed to be beneficial due to potential protection

319 against predators and other dangers during the vulnerable hours of sleep. This so-called sentinel

320 hypothesis developed out of the observation in Hadza hunter-gatherers of Tanzania that due to

321 variation in sleep phase timing all members of a group are rarely asleep simultaneously (Samson,

322 Crittenden, Mabulla, Mabulla, \& Nunn, 2017). Thus, it is possible that LTBS maintained

323 variation at this locus to promote chronotype diversity to mitigate the risks of sleeping.

326 (hg19.chr3:143636420-143740729) are associated with a range of risky behaviors and

327 educational attainment in both individual GWAS studies and the UK Biobank (Figure 5C). For

328 example, they are associated with automobile speeding propensity $(\mathrm{P}=4.4 \mathrm{E}-8)$ (Linnér, 2019)

329 and with educational attainment $(\mathrm{P}=2.4 \mathrm{E}-7)$ (Lee et al., 2018). The TSPs are also modestly

330 associated with variation in brain white matter microstructure (Anterior corona radiata mean

331 diusivities, $\mathrm{P}=1.96 \mathrm{E}-6$ ) (Zhao et al., 2019). Many of the variants in high LD with the TSPs in

332 this region have similar associations and overlap annotated regulatory regions: regulatory open

333 chromatin (rs10662845 and rs6766439), promoter (rs1898263, rs1992094, rs4431106,

334 rs7631704, rs7651567, and rs7653431), and CTCF binding sites (rs7628282, rs7650239,

335 rs7650332, rs9840519, rs9840971, rs9878070, and rs9840157). Furthermore, the TSPs (and 159

336 high LD variants) are significant eQTLs $(\mathrm{P} \leq 1 \mathrm{E}-5)$ for the gene DIPK2A (C3orf58) across four

337 GTEx tissues (small intestine terminal ileum, transformed fibroblasts, skin from the lower leg,

338 and suprapubic skin). DIPK2A has not been comprehensively functionally characterized, but it 
contains a protein kinase domain and is broadly expressed, including in the developing and adult

340 brain. Deletion of this gene has been linked to autism, and its expression is responsive to

341 neuronal activity (Morrow et al., 2008). Associations with behavioral and cognitive traits must

342 be interpreted with caution as these traits are very challenging to quantify and strongly

343 influenced by social factors that may vary with other characteristics. Nonetheless, these

344 associations point to an influence of the TSPs on behaviors relevant to risk tolerance. Thus, it is

345 possible that maintaining a diversity of risk tolerance in human and chimpanzee populations has

346 been beneficial.

348 Discussion

349 In this study we aimed to characterize the function of TSPs with evidence of LTBS. These

350 variants have a deep ancestry in the common ancestor between humans and chimpanzees, and

351 have persisted in the genomes of both species for millions of years. Due to the persistence of

352 these polymorphisms over such long periods, they are likely under balancing selection and have

353 functional effects that influence fitness (De Filippo et al., 2016), yet the majority of the TSPs

354 previously identified do not have known functions. To address this challenge, we identified

355 functional annotations for 114 out of 125 uncharacterized TSP regions with the help of newly

356 developed genomic annotation tools (Figure 2).

Our results show that TSPs likely maintained by LTBS have diverse functions beyond

358 enabling a flexible immune response to pathogens. This is consistent with several recent studies

359 of balancing selection over shorter timescales that have identified regions with functions outside

360 the immune system (Bitarello et al., 2018; Sato \& Kawata, 2018; Siewert \& Voight, 2017;

361 Viscardi et al., 2018). 
The associations we identify suggest possible behavioral, neurological, and other traits

363 that may have driven LTBS. In particular, our results provide support and candidate loci for

364 previous hypotheses about the need for neurological and behavioral diversity in populations. For

365 example, the chronotype association supports the sentinel hypothesis that variability in sleep

366 patterns is the result of natural selection acting to reduce vulnerability of groups while members

367 sleep at night (Samson et al., 2017). Similarly, selection has recently been shown to act on risk-

368 taking behavior in anole lizards (Lapiedra, Schoener, Leal, Losos, \& Kolbe, 2018). Thus, our

369 identification of an association between a TSP and human risk-taking behavior (Figure 4C)

370 suggests that LTBS may have maintained genetic variants that contribute to variation in risk

371 taking behavior in humans and chimpanzees. A gene with evidence of eQTL in this region

372 (C3orf58) encodes for a protein kinase and has been associated with autism and other

373 neurological disorders (Dudkiewicz, Lenart, \& Pawłowski, 2013), adding further evidence of

374 possible neurological drivers to LTBS.

Our results also raise the intriguing possibility that variants that modulate urate levels

376 have been under LTBS. Uricase, the enzyme that metabolizes uric acid into an easily excreted

377 water-soluble form in most mammals, has been lost in great apes. This gene was disabled by a

378 series of mutations that slowly decreased activity over primate evolution, increasing the levels of

379 uric acid in blood (Kratzer et al., 2014). It has been hypothesized that this loss of uricase activity

380 was driven by increase fructose in primate diets due to fruit eating (Johnson et al., 2009; Kratzer

381 et al., 2014). It has also been proposed that high levels of uric acid, a potent antioxidant, played

382 an important role in the evolution of intelligence, acting as antioxidant in the brain (Álvarez-

383 Lario \& Macarrón-Vicente, 2010). However, as reflected in the associations with this locus,

384 elevated uric acid levels contribute to many common diseases in modern humans, including 
chronic hypertension, cardiovascular disease, kidney and liver diseases, metabolic syndrome, diabetes, and obesity (Gustafsson \& Unwin, 2013). This suggests potential functional tradeoffs at this locus. However, we emphasize that proving the environmental drivers of past selection is challenging. Some of the phenotype associations we discovered may reflect manifestations of variation on traits in modern environments that could not be long-term drivers of balancing selection. As an extreme example, influence on smoking behavior could not have been the cause of LTBS given the relatively recent wide availability of alcohol and nicotine. Though we note that there is some evidence of ethanol consumption in chimpanzees (Hockings et al., 2015). Even

394 if they reflect modern environments, these associations provide hints about possible behavioral,

395 neurological, or other traits that may have driven LTBS. For instance, plant chemicals can hijack

396 reward systems in the brain that motivate repetition and learning (U.S. Department of Health \&

397 Human Services, 2016). The same systems that influence these action and consequently

398 reproductive fitness potentially created a byproduct of excessive seeking of dopamine or other 399 reward chemicals.

400 There are several caveats to our work. First, even with recent growth of genetic and 401 phenotypic databases, our knowledge of the functions of most regions of the genome is sparse.

402 Thus, failure to observe a functional association does not imply that a region does not have an 403 important function. Second, the genome- and phenome-wide association tools we used are 404 limited to the samples that have been analyzed; available data do not represent the full scope of 405 human variation. Most of the individuals analyzed in available genetic association studies are of 406 European ancestry. Variant functions and the ability to detect associations vary across human 407 populations; however, we anticipate that TSPs should have functional effects across populations, 
408 unless modern environments have masked the pressure driving LTBS. Third, even in PheWAS, a

409 limited number of phenotypes have been quantified across individuals, and these studies are

410 focused on a subset of clinically relevant traits rather evolutionarily relevant traits. Fourth, in

411 some analyses, we considered annotations based on trait associations with variants in high LD ( $\mathrm{r}^{2}$

$412>0.8$ ) with TSPs. This could potentially introduce false positives if the variant also tags a

413 different causal non-TSP variant. Given the long-term selection on TSPs, they are strong

414 candidates for casual variants, but functional studies are needed to confirm these statistical

415 associations. Finally, our analyses have focused on the human context; due to lack of functional

416 data, it is not possible to explore the function of TSPs in chimpanzees. Nonetheless, we feel that

417 our integration of genome-scale annotations and biobank data highlight the diversity of functions

418 associated with LTBS.

419 In conclusion, we assign putative functions for TSP regions that likely persisted due to

420 balancing selection dating back to at least the common ancestor of humans and chimpanzees.

421 These annotations expand beyond immune functions to traits relevant to behavior, cognition, and

422 body shape. Notably, we also find that most regions with multiple TSPs overlap gene regulatory

423 annotations suggesting LTBS on gene expression levels. As methods improve for quantifying the

424 effects of variants on gene regulation in different tissues and how these relate to organism-level

425 phenotypes, we anticipate deeper mechanistic understanding of the functions and potential

426 evolutionary pressures on these regions. 
430 The initial set of 263 TSPs from 125 regions analyzed in this study was published by Leffler et

431 al. (2013). The set is composed of trans-species polymorphisms that are shared between 51

432 Yoruba individuals in the 1000 Genomes Pilot 1 and 10 chimpanzees from the PanMap project.

433 Each SNP is part of a region composed of two or more TSPs within $4 \mathrm{~kb}$ of each other, where the

434 TMRCA between polymorphisms is deeper than the speciation event between humans and

435 chimpanzees.

436 To increase our ability to identify annotations in each locus, the dataset was expanded to

437 include variants in high $\mathrm{LD}$ (threshold $\mathrm{R}^{2}=0.8$ ) with each of the TSPs as is common in

438 association studies. We computed linkage disequilibrium with the TSP variants from 1000

439 Genomes Project Phase 3 data using rAggr a web tool developed by the University of Southern

440 California (http://raggr.usc.edu). We considered LD in African, East Asia, and European

441 populations. Variants with no reported RSID were excluded from the analysis. The dataset was

442 thus expanded by 9,996 SNPs in high LD with the TSPs for a total of 10,259 SNPs.

\section{Genome- and Phenome-wide associations}

445 The GWAS Catalog collects variant-trait associations from published genome-wide association

446 studies. The database is currently composed of more than 200,000 associations. We used the

447 GWAS Catalog version October 2020 to find functional associations for the LTBS variants. The

448 search was done using the BEDTools intersect function between the GWAS catalog and the LD-

449 expanded TSP dataset (Quinlan, 2014).

450 PheWAS is an analysis strategy built on top of medical records with information about

451 patient phenotypes and associated variants. The geneAtlas catalog

452 (http://geneatlas.roslin.ed.ac.uk/phewas/) takes advantage of the data provided by the UK 
453 Biobank cohort, which contains medically relevant data from nearly 500,000 British individuals

454 of European ancestry. This database contains 3 million variants in 778 traits. We matched our set

455 of variants against the geneAtlas database to search for traits associated with LTBS.

457 GTEx eQTL data

458 To evaluate potential gene regulatory effects of TSPs in non-coding regions, we analyzed data

459 from GTEx, a project developed to quantify the consequence of genetic variation on expression

460 at the tissue level (https://www.gtexportal.org/home/). The GTEx project v8 data have identified

461 eQTL across 50 tissues based on analyses of nearly 1,000 individuals to identify differential

462 expression through SNP variation. The intersection between the TSPs and LD SNPs and the

463 GTEx eQTL returned a large collection of TSP eQTL.

465 Other functional categories

466 We used ANNOVAR to identify variants overlapping protein coding regions. We used the

467 Ensembl Regulatory Build (Zerbino et al., 2015) to identify variants overlapping regions with

468 regulatory function. In all, 58 TSPs in 40 regions and 1334 LD SNPs in 114 regions overlapped

469 regulatory annotations.

471 Enrichment for overlap with regulatory regions

472 We used a permutation testing framework to calculate whether TSPs were more enriched for

473 overlap with regulatory regions than expected by chance (Benton, Talipineni, Kostka, \& Capra,

474 2019). We quantified the number of overlapping TSPs for each type of regulatory region (open

475 chromatin, promoter, enhancer, promoter-flanking, CTCF binding site, TF binding site). We then 
476 compared the observed TSP overlap to a null distribution of expected overlap generated by

477 randomly shuffling the regulatory regions 1000 times and obtained an empirical p-value. We

478 maintain the original length distribution and chromosome for shuffled regions and exclude all

479 ENCODE blacklist and gap regions (Kundaje, 2013).

\section{Declarations}

482

\section{Data Availability Statement}

484 The data underlying this article are available in the article and in its online supplementary

485 material.

\section{Competing interests}

488 The authors declare that they have no competing interests.

\section{$490 \quad$ Funding}

491 This work was supported by the National Institutes of Health [grant R35GM127087]; the

492 Burroughs-Wellcome Fund; and the National Institutes of Health [grant T32LM012412]. The

493 funders did not play any role in the study design, collection, analysis and interpretation of data, 494 or in writing the manuscript. 
Conceptualization: JAC; Methodology: KV, MLB, JAC; Investigation: KV, MLB, JAC; Writing

- Original Draft: KV, JAC; Writing - Review \& Editing: KV, MLB, JAC; Funding Acquisition:

JAC; Resources: JAC; Supervision: JAC.

\section{Acknowledgements}

502 We thank Evonne McArthur, David Rinker, and other members of the Capra Lab for helpful 503 comments on this work. This work was conducted in part using the resources of the Advanced

504 Computing Center for Research and Education at Vanderbilt University, Nashville, TN.

\section{REFERENCES}

507 Álvarez-Lario, B., \& Macarrón-Vicente, J. (2010). Uric acid and evolution. Rheumatology, 49(11), 2010-2015. https://doi.org/10.1093/rheumatology/keq204

Azevedo, L., Serrano, C., Amorim, A., \& Cooper, D. N. (2015). Trans-species polymorphism in humans and the great apes is generally maintained by balancing selection that modulates the host immune response. Human Genomics, 9(1). https://doi.org/10.1186/s40246-015-0043-1

Battivelli, E., Migraine, J., Lecossier, D., Yeni, P., Clavel, F., \& Hance, A. J. (2011). Gag

Benton, M. L., Talipineni, S. C., Kostka, D., \& Capra, J. A. (2019). Genome-wide enhancer annotations differ significantly in genomic distribution, evolution, and function. $B M C$ Genomics, 20(1), 1-22. https://doi.org/10.1186/s12864-019-5779-x

519 Bitarello, B. D., De Filippo, C., Teixeira, J. C., Schmidt, J. M., Kleinert, P., Meyer, D., \& 
Andres, A. M. (2018). Signatures of long-term balancing selection in human genomes.

Bush, W. S., Oetjens, M. T., \& Crawford, D. C. (2016, March 1). Unravelling the human genome-phenome relationship using phenome-wide association studies. Nature Reviews Genetics. Nature Publishing Group. https://doi.org/10.1038/nrg.2015.36

Cagliani, R., Fumagalli, M., Biasin, M., Piacentini, L., Riva, S., Pozzoli, U., ... Sironi, M. (2010). Long-term balancing selection maintains trans-specific polymorphisms in the human TRIM5 gene. Human Genetics, 128(6), 577-588. https://doi.org/10.1007/s00439-

Cagliani, R., Guerini, F. R., Fumagalli, M., Riva, S., Agliardi, C., Galimberti, D., ... Sironi, M. (2012). A trans-specific polymorphism in ZC3HAV1 is maintained by long-standing genotype of rs2941484 in the human HNF4G gene is associated with hyperuricemia in Chinese Han men. Oncotarget, 8(16), 26918-26926. balancing selection and may confer susceptibility to multiple sclerosis. Molecular Biology https://doi.org/10.18632/oncotarget.15851

Cheng, X., \& DeGiorgio, M. (2019). Detection of Shared Balancing Selection in the Absence of Trans-Species Polymorphism. Molecular Biology and Evolution, 36(1), 177-199. https://doi.org/10.1093/molbev/msy202

De Filippo, C., Key, F. M., Ghirotto, S., Benazzo, A., Meneu, J. R., Weihmann, A., ... Andrés, A. M. (2016). Recent Selection Changes in Human Genes under Long-Term Balancing Selection. Molecular Biology and Evolution, 33(6), 1435-1447. 
https://doi.org/10.1093/molbev/msw023

544 DeGiorgio, M., Lohmueller, K. E., \& Nielsen, R. (2014). A Model-Based Approach for Identifying Signatures of Ancient Balancing Selection in Genetic Data. PLoS Genetics,

Dudkiewicz, M., Lenart, A., \& Pawłowski, K. (2013). A Novel Predicted Calcium-Regulated 10(8). https://doi.org/10.1371/journal.pgen.1004561

Ganser-Pornillos, B. K., \& Pornillos, O. (2019, September 1). Restriction of HIV-1 and other retroviruses by TRIM5. Nature Reviews Microbiology. Nature Publishing Group. https://doi.org/10.1038/s41579-019-0225-2

Gustafsson, D., \& Unwin, R. (2013). The pathophysiology of hyperuricaemia and its possible relationship to cardiovascular disease, morbidity and mortality. BMC Nephrology.

Hockings, K. J., Bryson-Morrison, N., Carvalho, S., Fujisawa, M., Humle, T., McGrew, W. C., ... Matsuzawa, T. (2015). Tools to tipple: Ethanol ingestion by wild chimpanzees using leaf-sponges. Royal Society Open Science, 2(6). https://doi.org/10.1098/rsos.150150

Johnson, R. J., Sautin, Y. Y., Oliver, W. J., Roncal, C., Mu, W., Gabriela Sanchez-Lozada, L., ... 
individuals provides insights into circadian rhythms. Nature Communications, 10(343).

Kanai, M., Akiyama, M., Takahashi, A., Matoba, N., Momozawa, Y., Ikeda, M., ... Kamatani, Y. (2018). Genetic analysis of quantitative traits in the Japanese population links cell types to complex human diseases. Nature Genetics, 50(3), 390-400. https://doi.org/10.1038/s41588-018-0047-6

Key, F. M., Teixeira, J. C., de Filippo, C., \& Andrés, A. M. (2014). Advantageous diversity maintained by balancing selection in humans. Current Opinion in Genetics and

Kononoff, J., Kallupi, M., Kimbrough, A., Conlisk, D., de Guglielmo, G., \& George, O. (2018). Systemic and intra-habenular activation of the orphan $\mathrm{G}$ protein-coupled receptor GPR139 decreases compulsive-like alcohol drinking and hyperalgesia in alcohol-dependent rats.

Köttgen, A., Albrecht, E., Teumer, A., Vitart, V., Krumsiek, J., Hundertmark, C., ... Gieger, C. (2013). Genome-wide association analyses identify 18 new loci associated with serum urate concentrations. Nature Genetics, 45(2), 145-154. https://doi.org/10.1038/ng.2500

Kratzer, J. T., Lanaspa, M. A., Murphy, M. N., Cicerchi, C., Graves, C. L., Tipton, P. A., ... genome. Retrieved from 
yFreeze/jan2011/blacklists/hg19-blacklist-README.pdf,

Lapiedra, O., Schoener, T. W., Leal, M., Losos, J. B., \& Kolbe, J. J. (2018). Predator-driven natural selection on risk-taking behavior in anole lizards. Science, 360(6392), 1017-1020. https://doi.org/10.1126/science.aap9289

594 Lee, J. J., Wedow, R., Okbay, A., Kong, E., Maghzian, O., Zacher, M., ... Turley, P. (2018). educational attainment in 1.1 million individuals. Nature Genetics, 50(8), 1112-1121.

Leffler, E. M., Gao, Z., Pfeifer, S., Ségurel, L., Auton, A., Venn, O., ... Przeworski, M. (2013). Multiple instances of ancient balancing selection shared between humans and chimpanzees. over 1 million individuals identify hundreds of loci and shared genetic influences. Nature Genetics, 51(2), 245-257. https://doi.org/10.1038/s41588-018-0309-3

Mao, R., Nie, H., Cai, D., Zhang, J., Liu, H., Yan, R., ... Guo, H. (2013). Inhibition of Hepatitis https://doi.org/10.1371/journal.ppat.1003494 321(5886), 218-223. https://doi.org/10.1126/science.1157657 D. (2011). LocusZoom: Regional visualization of genome-wide association scan results. In 
Bioinformatics (Vol. 27, pp. 2336-2337). Oxford University Press.

614 Quinlan, A. R. (2014). BEDTools: The Swiss-Army tool for genome feature analysis. Current 615 Protocols in Bioinformatics, 47(1), 11-12. https://doi.org/10.1002/0471250953.bi1112s47

616 Samson, D. R., Crittenden, A. N., Mabulla, I. A., Mabulla, A. Z. P., \& Nunn, C. L. (2017).

617 Chronotype variation drives night-time sentinel-like behaviour in hunter-gatherers. Proceedings of the Royal Society B: Biological Sciences, 284(20170967). https://doi.org/10.1098/rspb.2017.0967

Sanchez-Roige, S., Palmer, A. A., Fontanillas, P., Elson, S. L., Adams, M. J., Howard, D. M., ... Wilson, C. H. (2019). Genome-wide association study meta-analysis of the alcohol use disorders identification test (AUDIT) in two population-based cohorts. American Journal of associated with psychiatric disorders and human-unique personality traits. Evolution Psychiatry, 176(2), 107-118. https://doi.org/10.1176/appi.ajp.2018.18040369

Ségurel, L., Thompson, E. E., Flutre, T., Lovstad, J., Venkat, A., Margulis, S. W., ... Przeworski, M. (2012). The ABO blood group is a trans-species polymorphism in primates. Proceedings

Siewert, K. M., \& Voight, B. F. (2017). Detecting long-term balancing selection using allele frequency correlation. Molecular Biology and Evolution, 34(11), 2996-3005. of the National Academy of Sciences of the United States of America, 109(45), 18493https://doi.org/10.1093/molbev/msx209

Siewert, K. M., \& Voight, B. F. (2020). BetaScan2: Standardized Statistics to Detect Balancing 
Selection Utilizing Substitution Data. Genome Biology and Evolution, 12(2), 3873-3877. https://doi.org/10.1093/gbe/evaa013

637 Stotz, M., Szkandera, J., Seidel, J., Stojakovic, T., Samonigg, H., Reitz, D., ... Pichler, M.

638 (2014). Evaluation of uric acid as a prognostic blood-based marker in a large cohort of pancreatic cancer patients. $P L O S O N E, 9(8)$. https://doi.org/10.1371/journal.pone.0104730

640 Teixeira, J. C., De Filippo, C., Weihmann, A., Meneu, J. R., Racimo, F., Dannemann, M., ... Andrés, A. M. (2015). Long-term balancing selection in LAD1 maintains a missense transspecies polymorphism in humans, chimpanzees, and bonobos. Molecular Biology and Evolution, 32(5), 1186-1196. https://doi.org/10.1093/molbev/msv007

Tin, A., Marten, J., Halperin Kuhns, V. L., Li, Y., Wuttke, M., Kirsten, H., ... Köttgen, A. (2019). Target genes, variants, tissues and transcriptional pathways influencing human

Todorova, T., Bock, F. J., \& Chang, P. (2015, June 1). Poly(ADP-ribose) polymerase-13 and serum urate levels. Nature Genetics, 51(10), 1459-1474. https://doi.org/10.1038/s41588substance use, misuse, and addiction. Facing Addiction in America: The Surgeon General's Report on Alcohol, Drugs, and Health.

654 Viscardi, L. H., Paixão-Côrtes, V. R., Comas, D., Salzano, F. M., Rovaris, D., Bau, C. D., ... 655 Bortolini, M. C. (2018). Searching for ancient balanced polymorphisms shared between 656 Neanderthals and modern humans. Genetics and Molecular Biology, 41(1), 67-81. https://doi.org/10.1590/1678-4685-gmb-2017-0308 
658 Zerbino, D. R., Wilder, S. P., Johnson, N., Juettemann, T., \& Flicek, P. R. (2015). The Ensembl

659 Regulatory Build. Genome Biology, 16(1). https://doi.org/10.1186/s13059-015-0621-5

660 Zhao, B., Zhang, J., Ibrahim, J. G., Luo, T., Santelli, R. C., Li, Y., ... Zhu, H. (2019). Large-

661 scale GWAS reveals genetic architecture of brain white matter microstructure and genetic

662 overlap with cognitive and mental health traits $(\mathrm{n}=17,706)$. Molecular Psychiatry.

663 https://doi.org/10.1038/s41380-019-0569-z

664 